\title{
Dynamics and thermodynamics of water
}

\author{
Pradeep Kumar $^{1,2}$, Giancarlo Franzese ${ }^{3}$ and H Eugene Stanley ${ }^{1}$ \\ ${ }^{1}$ Center for Studies in Physics and Biology, Rockefeller University, New York, \\ NY 10021, USA \\ ${ }^{2}$ Center for Polymer Studies and Department of Physics, Boston University, \\ Boston, MA 02215, USA \\ ${ }^{3}$ Departament de Física Fonamental, Universitat de Barcelona, Diagonal 647, \\ Barcelona 08028, Spain \\ E-mail: pradeep.kumar@ rockefeller.edu,gfranzese@ub.edu and hes@bu.edu
}

Received 10 March 2008

Published 29 May 2008

Online at stacks.iop.org/JPhysCM/20/244114

\begin{abstract}
On decreasing the temperature $T$, the correlation time $\tau$ of supercooled water displays a dynamic crossover from non-Arrhenius dynamics (with $T$-dependent activation energy) at high $T$ to Arrhenius dynamics (with constant activation energy) at low $T$. Simulations for water models show that this crossover occurs at the locus of maximum isobaric specific heat in the pressure-temperature $(P-T)$ plane. Results of simulations show also that at this locus there is a sharp change of local structure: more tetrahedral below the locus, and less tetrahedral above it. Furthermore, in water solutions with proteins or DNA, simulations show that in correspondence with this locus there is a crossover in the dynamics of the biomolecules, a phenomenon commonly known as the protein glass transition.

To clarify the relation of the dynamic crossover with the thermodynamics of water, we study the dynamics of a cell model of water which can be tuned to exhibit: (1) a first-order phase transition line that separates the liquids of high and low densities at low temperatures; this phase transition line terminates at a liquid-liquid critical point (LLCP), from which departs the Widom line $T_{\mathrm{W}}(P)$, i.e. the line of maximum isobaric specific heat in the $P-T$ plane;

(2) the singularity-free (SF) scenario, under which the system exhibits water-like anomalies but with no finite temperature liquid-liquid critical point.

We find that the dynamic crossover is present in both the LLCP and the SF cases. Moreover, on the basis of the study of the probability $p_{\mathrm{B}}$ of forming a bond, we propose and verify a relation between dynamics and thermodynamics that is able to show how the crossover is a consequence of a local relaxation process associated with breaking a bond and reorienting the molecule. We further find a distinct difference in pressure dependence of the dynamic crossover between the LLCP and SF scenarios, which may help in resolving which of the scenarios correctly explains the anomalous behavior of water.
\end{abstract}

(Some figures in this article are in colour only in the electronic version)

\section{Introduction}

Our life depends on water, yet many unique properties of water still present a puzzle for which different interpretations have been proposed in the past. Water has more than sixty anomalies, such as the increase of density upon increasing temperature or its extraordinary large capacity of absorbing heat, essential for regulating our body temperature. Its heat capacity, contrarily to most of the liquids, increases at low temperatures, where other anomalies appear. For example, water can stay liquid at very low temperature in a metastable supercooled state: down to $-47^{\circ} \mathrm{C}$ in plants and $-92^{\circ} \mathrm{C}$ in laboratory at a pressure of $2 \mathrm{kbars}$ [1].

In the following we briefly summarize few of the thermodynamic and dynamic anomalies of water.

\subsection{Thermodynamic anomalies of liquid water}

1.1.1. Density anomaly. The density anomaly is perhaps the oldest known puzzling behavior of water [2]. Unlike 

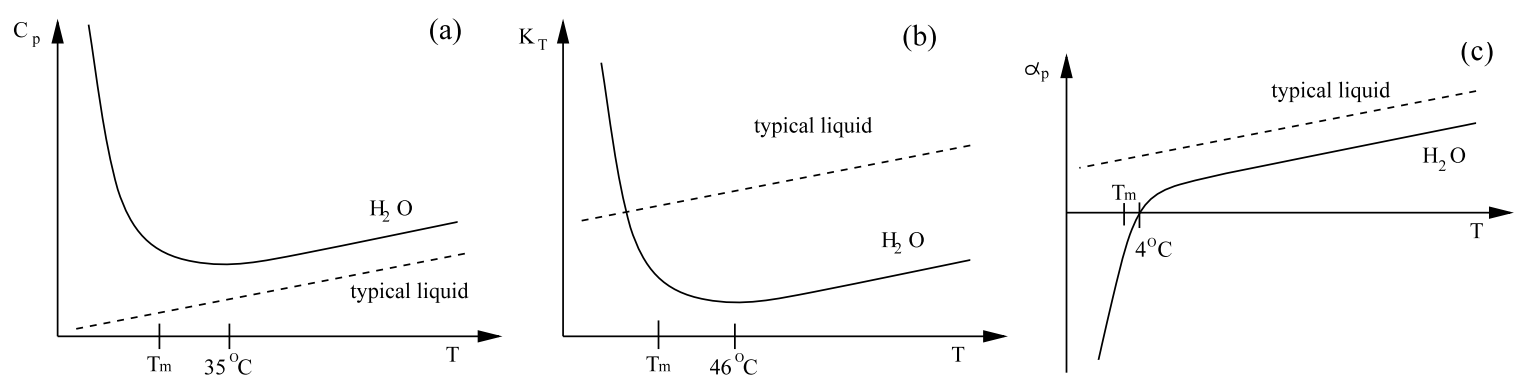

Figure 1. Schematic representation of response functions (a) $C_{P}$ (b) $K_{T}$ and (c) $\alpha_{P}$ of liquid water as a function of temperature $T$. The behavior of a normal liquid is shown as the dashed curve.

other simple liquids which expand upon heating, water expand upon cooling below $277 \mathrm{~K}$ at ambient pressure. It is for this anomaly that ice floats on water and fishes can survive in warm waters below a layer of ice at temperatures well below $0{ }^{\circ} \mathrm{C}$. The temperature of maximum density, $T_{\mathrm{MD}}$, decreases as the pressure is increased and disappears above $\approx 200 \mathrm{MPa}$. In the $(T, P)$ plane, the region below the locus of $T_{\mathrm{MD}}$ is where the density anomaly occurs. Computer simulations of different models of water [3] find the $T_{\mathrm{MD}}$ as in experiments. Recent experiments on water confined in nanopores $[4,5]$ show that below approximately $210 \mathrm{~K}$ the supercooled liquid exhibits a density minimum and recovers a normal behavior in density.

1.1.2. Specific heat. A schematic isobaric heat capacity $C_{P}$ for liquid water at atmospheric pressure is shown in figure 1(a). $C_{P}$ is a measure of how enthalpy $H$ changes with $T$, at constant $P$, and is related to entropy fluctuations $\left\langle(\Delta S)^{2}\right\rangle[6,7]$ as:

$$
C_{P} \equiv\left(\frac{\mathrm{d} H}{\mathrm{~d} T}\right)_{P}=T\left(\frac{\partial S}{\partial T}\right)_{P}=\frac{\left\langle(\Delta S)^{2}\right\rangle}{k_{\mathrm{B}}}
$$

where $S$ is the entropy and $k_{\mathrm{B}}$ is the Boltzmann constant. Since any thermal fluctuation should decrease with decreasing temperature, one would expect the same behavior for $C_{P}$. Instead, for the case of water it increases sharply as the temperature is decreased below approximately $330 \mathrm{~K} . C_{P}$ seems to diverge as a power law at about $228 \mathrm{~K}$ [8].

1.1.3. Isothermal compressibility. A schematic isothermal compressibility $K_{T}$ for water is shown in figure 1(b). $K_{T}$ is the measure of volume fluctuations $\left\langle(\Delta V)^{2}\right\rangle$ :

$$
K_{T} \equiv-\frac{1}{V}\left(\frac{\partial V}{\partial P}\right)_{T}=\frac{\left\langle(\Delta V)^{2}\right\rangle}{k_{\mathrm{B}} T V} .
$$

Intuitively $K_{T}$ should decrease upon decreasing the temperature. In the case of water, instead, it increases like $C_{P}$ and seems to diverge with a power law at about $228 \mathrm{~K}$ [8].

1.1.4. Coefficient of thermal expansion. Coefficient of thermal expansion $\alpha_{P}$ is the measure of cross fluctuations of volume and entropy $\langle\Delta V \Delta S\rangle$ :

$$
\alpha_{P} \equiv \frac{1}{V}\left(\frac{\partial V}{\partial T}\right)_{P}=\frac{P}{k_{\mathrm{B}}^{2} T}\langle\Delta V \Delta S\rangle .
$$

$\alpha_{P}$ is positive for normal liquids (figure 1(c)). Instead, in the case of water it becomes negative at the temperature of maximum density $T_{\mathrm{MD}}$, indicating that for $T<T_{\mathrm{MD}}$ the entropy decreases when the volume increases. In experiments, like other response functions, $\alpha_{P}$ also seems to diverge with a power law at about $228 \mathrm{~K}$ [8]. Dashed curves in figures 1(a)(c) are the schematic representations of the behavior of normal liquids for a comparison.

1.1.5. Diffusion anomaly. The dynamics of simple liquids becomes slower upon pressurizing. Instead, the dynamics of water becomes faster as the pressure is increased reaching a maximum at a constant temperature [9]. The region of this dynamic anomaly includes the region of density anomaly in the $(T, P)$ plane [10]. Computer simulations of different models of water recover the experimental results [11-13]. They show that the diffusion constant $D$ decreases for decreasing $P$, until it reaches a minimum value at some negative pressure below which the normal behavior is recovered [13-17]. The anomalous increase of diffusion upon pressurizing is attributed to breaking of hydrogen bonds. As the pressure is increased more and more hydrogen bonds are broken, making the water molecules diffuse free from their neighbors and hence the increase of diffusion.

1.1.6. Non-Arrhenius to Arrhenius dynamic crossover at low temperatures. Liquids with relaxation times that are an exponential function of $1 / T$ are said to have an Arrhenius (or activated) behavior, while those whose relaxation times follow a different function of $1 / T$ are said to have a non-Arrhenius behavior. If, instead of the relaxation time of some specific degree of freedom, the viscosity is used to characterize the dynamics, then the variation of viscosity as an exponential function of $1 / T$ is called 'strong' behavior, while a different function of $1 / T$ is called 'fragile' behavior [18].

Normal liquids show only one of the two behaviors: they are or Arrhenius or non-Arrhenius, as well as they are strong or fragile. Water, instead, is anomalous also in this respect, because it shows a crossover from a non-Arrhenius behavior at high $T$ to an Arrhenius behavior at low $T$ in the relaxations times, as well as a (high- $T$ ) fragile to (low- $T$ ) strong crossover [18].

The investigation on this anomaly has received a recent boost thanks to the experiments on water confined in 


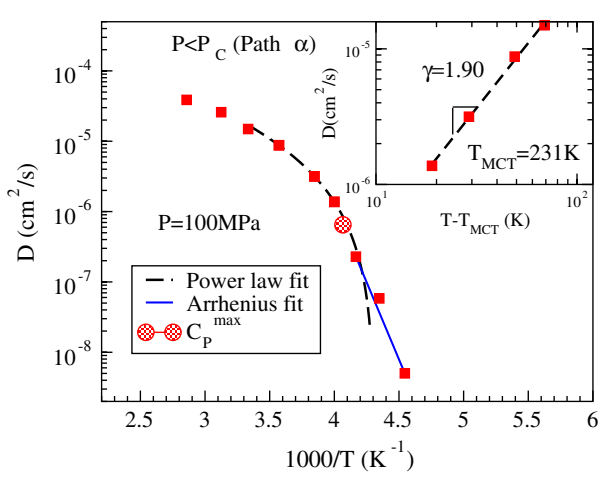

Figure 2. Non-Arrhenius to Arrhenius crossover in the dynamics of TIP5P model of water. The diffusion constant $D$ as a function of $1 / T$ [34]. At high $T$, the temperature dependence of $D$ can be fit with a power law and, at low $T, D$ becomes Arrhenius. Inset: $D$ behaves as a power low of $T_{\mathrm{MCT}}$, where $T_{\mathrm{MCT}}$ is a fitting parameter (mode coupling theory temperature).

nanostructures [19-21], water hydrating biomolecules [22], and computer simulations [19, 23-33]. Both experiments and simulations show a non-Arrhenius to Arrhenius crossover in relaxation times and diffusion constant $D$ (figure 2) [34] whose relation with the bulk-water behavior is under investigation [18].

One possible interpretation of the anomalous properties of water is the presence of a hypothesized liquid-liquid critical point (LLCP) $C^{\prime}[35]$ in the supercooled phase. However, as we discuss in the next sections, this is not the only possible interpretation.

\subsection{Interpretations of the anomalies of water}

Many of the anomalies of water can be reproduced by several mechanisms. For example, it has been shown that isotropic interactions of models with a LLCP can display the anomalies we described above [36-38], with the same hierarchy observed in water [39-41]. However, it has been shown also that not any isotropic potential with a LLCP displays water anomalies [42], questioning which details of the interaction are relevant to get the complete picture. Usually all these results are analyzed in the framework of two main interpretations, although other hypothesis are under discussion [18].

1.2.1. The liquid-liquid critical point (LLCP) scenario. The experimental results discussed in the previous section can be interpreted in a consistent way by hypothesizing the presence of a critical point between two metastable fluid phases for supercooled water. This critical point is the terminus of a phase transition line that separates a low-density liquid (LDL) and a high-density liquid (HDL). This liquid-liquid critical point (LLCP) gives rise to the Widom line $T_{\mathrm{W}}(P)$ in the supercritical liquid region, defined as the locus where different response functions, such $C_{P}, K_{T}$ or $\alpha_{P}$, have a maximum [34, 43]. The correlation length increases on approaching $C^{\prime}$ along the Widom line and diverges at $C^{\prime}$.

Since the experiments on bulk liquid water cannot be performed below the homogeneous nucleation temperature
$T_{\mathrm{H}}^{\mathrm{bulk}} \approx-38^{\circ} \mathrm{C}$, where the crystal formation is inevitable, it is not possible to test whether the seeming divergence of response functions at low temperatures is indeed a divergence or something else. Xu et al [34] studied different models of water and found that $C_{P}, K_{T}$, and $\alpha_{P}$ indeed increase sharply as the temperature is increased, however instead of diverging at low temperatures they have an extremum. They further found that the maxima of these response functions increase as the pressure is increased and ultimately diverge [34]. This behavior of the response functions is consistent with hypothesis of a negatively sloped liquid-liquid phase coexistence line ending at a critical point $[34,22,44]$.

\subsubsection{The singularity-free $(S F)$ scenario. Another} thermodynamically consistent interpretation of the water anomalies is known as 'singularity-free' scenario (SF) [45]. Using a cell model of water, it was proposed that the rise in response functions upon cooling can be described entirely by the anticorrelation in volume and entropy fluctuations with no singularity, differently from the case of LLCP scenario. The SF scenario predicts, as well as the LLCP scenario [34, 44, 46], a maximum in the response functions such as $K_{T}, \alpha_{P}$ or $C_{P}$, but, differently from the LLCP, only the maxima of $K_{T}$ and $\alpha_{P}$ increase upon increasing $P$, while the maxima of $C_{P}$ do not change in height $[47,46]$.

Until recently [46], this was the only difference, between the SF and the LLCP scenario, that was predicted above $T_{\mathrm{H}}^{\mathrm{bulk}}$, the temperature of the inaccessible region possibly hiding the critical point of the LLCP scenario. In the attempt to explore the region below $T_{\mathrm{H}}^{\text {bulk }}$, and clarify the low- $T$ phase diagram of water, many investigations have been done recently on confined water and in water hydrating macromolecules $[4,5,19,20,22,21,48]$. In these cases, indeed, the crystallization can be, at least partially, avoided even for $T<T_{\mathrm{H}}^{\mathrm{bulk}}$. However, the interpretation of the results can be controversial $[34,21]$. For this reason simulations on confined and bulk water can help in clarifying the experimental data.

\subsection{Low temperature dynamics of hydrated biomolecules}

Recently it has been hypothesized, on the base of molecular dynamics (MD) simulations [43], that a dynamic crossover observed in biomolecules, and called biomolecules glass transition [22, 49-61], is related to the liquid-liquid phase transition. Specifically, Kumar et al [43] studied the dynamic and thermodynamic behavior of TIP5P water hydrating (i) an orthorhombic form of hen egg-white lysozyme [63] and (ii) a Dickerson dodecamer DNA [64] at constant pressure $P=$ $1 \mathrm{~atm}$, several constant temperatures $T$, and constant number of water molecules $N$. They calculated the mean square fluctuations $\left\langle x^{2}\right\rangle$ of the biomolecules from the equilibrated configurations, averaged over $1 \mathrm{~ns}$. They found that the temperature dependence of $\left\langle x^{2}\right\rangle$ shows a crossover at $T_{\mathrm{p}} \approx$ $245 \mathrm{~K}$, for both lysozyme (figure 3(a)) and DNA (figure 3(b)).

Kumar et al next calculated $C_{P}$ by numerical differentiation of the total enthalpy of the system (protein and water) by fitting the simulation data for enthalpy with a fifthorder polynomial, and then taking the derivative with respect 

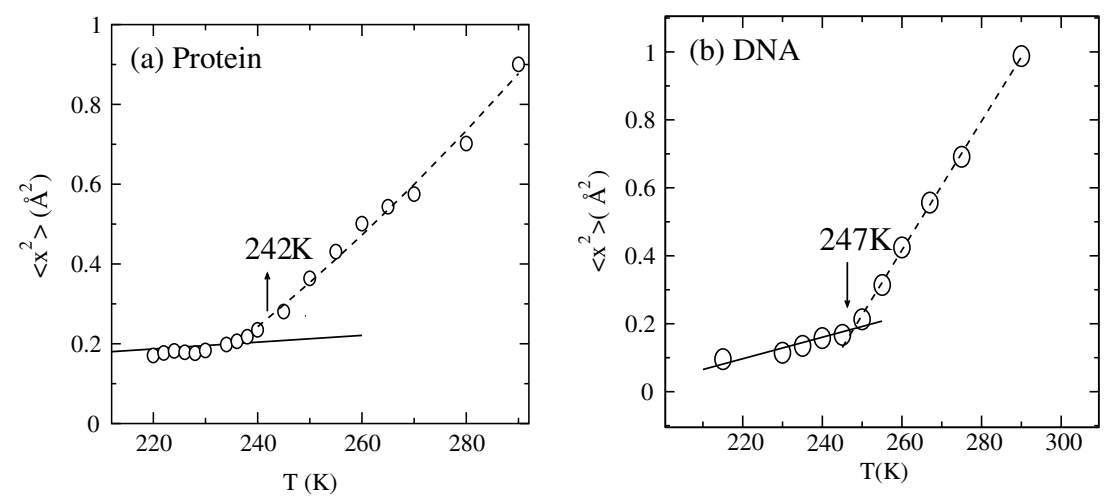

Figure 3. Mean square fluctuation of (a) lysozyme, and (b) DNA showing that there is a transition around $T_{\mathrm{p}} \approx 242 \pm 10 \mathrm{~K}$ for lysozyme and around $T_{\mathrm{p}} \approx 247 \pm 10 \mathrm{~K}$ for DNA. For very low $T$ one would expect a linear increase of $\left\langle x^{2}\right\rangle$ with $T$, as a consequence of harmonic approximation for the motion of residues. At high $T$, the motion becomes non-harmonic and we fit the data by a polynomial. The dynamic crossover temperature $T_{\mathrm{p}}$ is determined from the crossing of the linear fit for low $T$ and the polynomial fit for high $T$. The error bars is estimated by changing the number of data points in the two fitting ranges.

to $T$. Figures $4(\mathrm{a})$ and (b) display maxima of $C_{P}(T)$ at $T_{\mathrm{W}} \approx 250 \pm 10 \mathrm{~K}$, as well as figures $4(\mathrm{c})$ and (d) show maxima of the derivative $|\mathrm{d} Q / \mathrm{d} T|$ of the local tetrahedral-order parameter $Q$ with respect to temperature at the same $T_{\mathrm{W}}$, while figures 4(e) and (f) display a dynamic crossover at $T_{\times}$for the diffusion constant of hydration water for both biomolecules.

The fact that at $T_{\mathrm{W}}$ both $C_{P}$ and $|\mathrm{d} Q / \mathrm{d} T|$ have a maximum is consistent with the observation that crossing the Widom line corresponds to a continuous but rapid transition of the properties of water from those resembling the properties of a local HDL structure for $T>T_{\mathrm{W}}(P)$ to those resembling the properties of a local LDL structure for $T<$ $T_{\mathrm{W}}(P)[34,20,43]$. A consequence is the expectation that the fluctuations of the protein residues in predominantly LDL-like water (more ordered and more rigid) just below the Widom line should be smaller than the fluctuations in predominantly HDLlike water (less ordered and less rigid) just above the Widom line.

This is, indeed, the case with $T_{\mathrm{p}} \approx T_{\mathrm{W}}$ suggesting the correlation between the changes in protein fluctuations and the hydration water thermodynamics. Furthermore, the fact that $T_{\mathrm{p}} \approx T_{\times}$suggests that it is indeed the changes in the properties of hydration water that are responsible for the changes in dynamics of the protein and DNA biomolecules.

\subsection{Possible interpretation of the hydration water results and the use of a tunable cell model for bulk water}

These results are in qualitative agreement with recent experiments on hydrated protein and DNA [22] which found the crossover in side-chain fluctuations at $T_{\mathrm{p}} \approx 225 \mathrm{~K}$ and are all consistent with the possibility that the protein glass transition is related to the Widom line and to the hypothesized LLCP [65]. However, another possibility is that the dynamic crossover in experiments, occurring at the maximum in $C_{P}$, is due to the SF mechanism, with no increase and divergence of the correlation length as predicted in the LLCP interpretation. For this reason we have analyzed the two hypothesis by means of a cell model that can reproduce both scenarios, the LLCP and the SF, depending on the value of a single parameter [66]. We looked for differences between the two cases, with the aim of clarifying which scenario is more suitable to describe the experiments.

One of advantages of this approach with respect to direct simulations of less schematic models, is that in the cell model the relation between the dynamics and the thermodynamics can be explicitly calculated and not only inferred by the numerical evidences, as in MD simulations. Another advantage is that, by tuning the cell model between the two scenarios, we understand which differences in the dynamics are related to the different thermodynamics of the two interpretations.

To reduce the complexity of the analysis we considered the case of bulk water [46]. We calculated the relation between dynamics and thermodynamics, showing that the dynamic crossover is a direct consequence of the structural change at the temperature of maximum $C_{P}$. We expressed the relevant free energy barrier for the local rearrangement of the molecules in terms of the probability $p_{\mathrm{B}}$ of forming bonds. By mean field calculations and Monte Carlo (MC) simulations we found that the variation of $p_{\mathrm{B}}$ is the largest at the locus of the maximum $C_{P}$, in both scenarios [46].

Nevertheless, we found a difference between the two scenarios. We studied the ratio between the activation energy in the Arrhenius regime and the crossover temperature and we found that this index increases upon increasing pressure in the LLCP scenario, while stays constant in the SF case [46]. Since this index can be measured in the supercooled phase of liquid water, it could provide the first observable quantity that allows to distinguish which scenario holds for the low temperature phase diagram of water.

\section{Hamiltonian model for water}

We consider a cell model that reproduces the fluid phase diagram of water and other tetrahedral network-forming liquids [66]. For sake of clarity, we focus on water to explain the motivation of the model. The model is based on the experimental observations that on decreasing $P$ at constant 

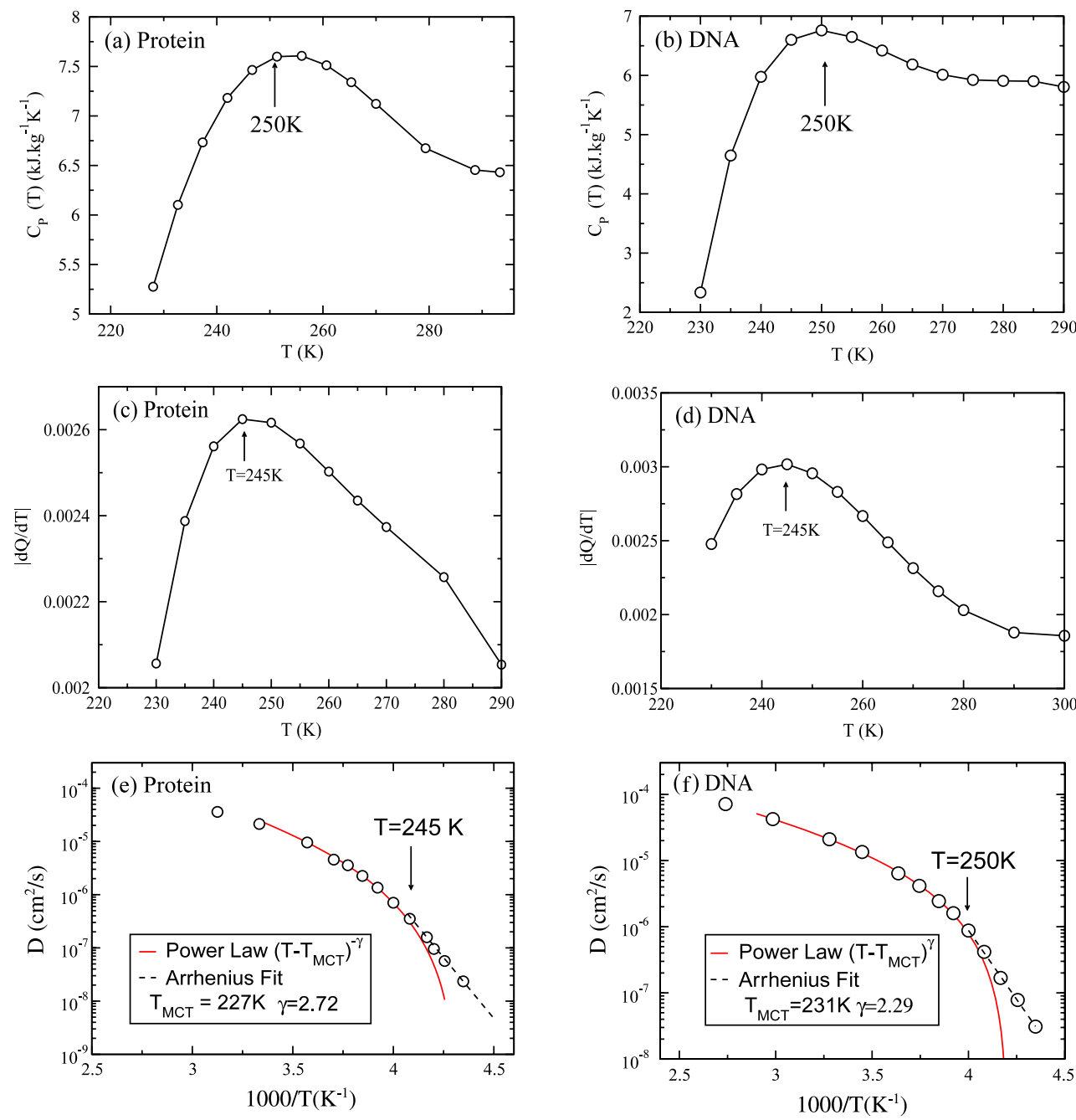

Figure 4. The specific heat of the combined system (a) lysozyme and water, and (b) DNA and water, display maxima at $T_{\mathrm{W}} \approx 250 \pm 10 \mathrm{~K}$ and $250 \pm 12 \mathrm{~K}$ respectively. Derivative $|\mathrm{d} Q / \mathrm{d} T|$ of the tetrahedral-order parameter for (c) lysozyme and (d) DNA hydration water, shows a maximum at $T_{\mathrm{W}}$ (Widom line temperature) suggesting that the rate of change of local tetrahedrality of hydration water has a maximum at $T_{\mathrm{W}}$. Diffusion constant of hydration water surrounding (e) lysozyme, and (f) DNA shows a dynamic transition from a power-law behavior to an Arrhenius behavior at $T_{\times} \approx 245 \pm 10 \mathrm{~K}$ for lysozyme and $T_{\times} \approx 250 \pm 10 \mathrm{~K}$ for DNA, around the same temperatures $T_{\mathrm{p}}$, where the behavior of $\left\langle x^{2}\right\rangle$ has a crossover, and $T_{\mathrm{W}}$, where $C_{P}$ and $|\mathrm{d} Q / \mathrm{d} T|$ have maxima.

$T$, or on decreasing $T$ at constant $P$, (i) water displays an increasing local tetrahedrality [67], (ii) the volume per molecule increases at sufficiently low $P$ or $T$, and (iii) the $\mathrm{O}-$ $\mathrm{O}-\mathrm{O}$ angular correlation increases [68], as in simulations [69].

The system is divided into cells $i \in[1, \ldots, N]$ on a regular square lattice, each containing a molecule with volume $v \equiv V / N$, where $V \geqslant N v_{0}$ is the total volume of the system, and $v_{0}$ is the hard-core volume of one molecule. The cell volume $v$ is a continuous variable that gives the mean distance $r \equiv v^{1 / d}$ between molecules in $d$ dimensions. The van der Waals attraction between the molecules is represented by a truncated Lennard-Jones potential with characteristic energy $\epsilon>0$

$$
U(r) \equiv \begin{cases}\infty & \text { for } r \leqslant R_{0} \\ \epsilon\left[\left(\frac{R_{0}}{r}\right)^{12}-\left(\frac{R_{0}}{r}\right)^{6}\right] & \text { for } r>R_{0},\end{cases}
$$

where $R_{0} \equiv v_{0}^{1 / d}$ is the hard-core distance [66].
Each molecule $i$ has four bond indices $\sigma_{i j} \in[1, \ldots, q]$, corresponding to the nearest-neighbor cells $j$. When two nearest-neighbor molecules have the facing $\sigma_{i j}$ and $\sigma_{j i}$ in the same relative orientation, they decrease the energy by a constant $J$, with $0<J<\epsilon$, and form a bond, e.g. a (non-bifurcated) hydrogen bond for water, or a ionic bond for $\mathrm{SiO}_{2}$. An alternative choice to represent the orientations of a molecule could be to use four continuous XY variables. See for example [70]. The choice $J<\epsilon$ guarantees that bonds are formed only in the liquid phase. The bond interaction is accounted for by a term in the Hamiltonian

$$
\mathcal{H}_{\mathrm{B}} \equiv-J \sum_{\langle i, j\rangle} \delta_{\sigma_{i j} \sigma_{j i}}
$$

where the sum is over nearest-neighbor cells, and $\delta_{a, b}=1$ if $a=b$ and $\delta_{a, b}=0$ otherwise.

The model assumes that the tetrahedral coordination number is preserved for all $P$ and $T$. For water at high $P$ 
and $T$ a more dense, collapsed and distorted, local structure with bifurcated hydrogen bonds (HB) is consistent with the experiments. Bifurcated HBs decrease the strength of the network and favor the HB breaking and re-formation. The model simplifies the situation by assuming that (a) only nonbifurcated, i.e. normal, HBs decrease the energy of the system and (b) the local density changes as function of the number of normal HBs, consistent with the observation [68] that at low $P$ and $T$ there is a better separation between the first neighbors and the second neighbors, favoring normal HBs and the tetrahedral order.

The density decrease for the temperature of maximum density $T<T_{\mathrm{MD}}(P)$ is represented by an average increase of the molar volume due to a more structured network. The total volume increases by an amount $v_{\mathrm{B}}>0$ for each bond formed $[45,66]$, and hence

$$
v=v^{\prime}+2 p_{\mathrm{B}} v_{\mathrm{B}},
$$

where $v^{\prime}$ is the molar volume without taking into account the bond. The increase of the intramolecular angular correlation is modeled by introducing an intramolecular (IM) interaction of energy $0<J_{\sigma}<J$,

$$
\mathcal{H}_{\mathrm{IM}} \equiv-J_{\sigma} \sum_{i} \sum_{(k, \ell)_{i}} \delta_{\sigma_{i k} \sigma_{i \ell}}
$$

where $\sum_{(k, \ell)_{i}}$ denotes the sum over the bond indices of the molecule $i$.

The total energy of the system is the sum of equations (4), (5) and (7). We perform mean field calculations and MC simulations in the NPT ensemble [71, 66] for a system with $J / \epsilon=0.5, J_{\sigma} / \epsilon=0.05$ and $J_{\sigma}=0, v_{\mathrm{B}} / v_{0}=$ $0.5, q=6$. We find that the model displays a critical point $C^{\prime}$ between two liquids at different density, as in the LLCP scenario. We study two square lattices with 900 and 3600 cells, and find no appreciable size effects.

For $J_{\sigma} \rightarrow 0$, mean field calculations and MC simulations show that $C^{\prime}$ disappears at $T=0$ [72]. For $J_{\sigma}=0$, the model coincides with the one studied by Sastry et al in [45], giving rise to the SF scenario.

\section{Liquid-liquid critical point (LLCP) scenario}

Below the $T_{\mathrm{MD}}$ line, in the supercooled region, the model displays a first-order phase transition between a LDL at low $P$ and $T$ and a HDL at high $P$ and $T$ along a line terminating in the liquid-liquid critical point $C^{\prime}$ [66] (figure 5(a)).

\subsection{Fluctuations in the supercritical region}

For $P<P_{C^{\prime}}$, the pressure of $C^{\prime}$, we find that the constant pressure specific heat $C_{P}(T)$ and thermal expansion coefficient $\left|\alpha_{P}\right|$ have maxima that move to lower $T$ as $P$ is increased (figures 7(a) and 5(b)). The loci of the maxima of $C_{P}(T)$ and $\left|\alpha_{P}(T)\right|$ merge close to $C^{\prime}$. The amplitudes of these maxima increase on approaching $C^{\prime}$. This is consistent with the expected divergence of the correlation length at $C^{\prime}$. The size of $\left|\alpha_{P}\right|^{\max }$ increases rapidly as $C^{\prime}$ is approached, while
$C_{P}^{\max }$ increases less rapidly (figure 7(a)). The Widom line $T_{\mathrm{W}}(P)$ coincides with the loci of $C_{P}^{\max }$ and $\left|\alpha_{P}\right|^{\max }$ close to $C^{\prime}[34,71]$. We choose $T_{\mathrm{W}}(P)$ to be the mid-point between $C_{P}^{\max }$ and $\left|\alpha_{P}\right|^{\max }$, with an error equal to the sum of the $C_{P}$ and $\left|\alpha_{P}\right|$ errors.

We find that $p_{\mathrm{B}}$ increases on decreasing $T$ (figure 5(c)), with $p_{\mathrm{B}} \simeq 0.8$ at $T_{\mathrm{W}}(P)$. The value of $p_{\mathrm{B}}\left(T_{\mathrm{W}}\right)$ weakly decreases for increasing $P$ and polynomial extrapolations to $C^{\prime}$ up to the fifth order lead to a value of $p_{\mathrm{B}}\left(P_{C^{\prime}}\right)=0.55 \pm$ 0.15 . Hence, close to $P_{C^{\prime}}$, on crossing $T_{\mathrm{W}}(P)$ there is a large variation from $p_{\mathrm{B}} \approx 1 / 2$, to $p_{\mathrm{B}}=1$. The mean field [71] calculation of $p_{\mathrm{B}}$ (figure 5(c)) compares well with simulations for $T>T_{\mathrm{W}}(P)$ and for $T \ll T_{\mathrm{W}}(P)$, with increasing discrepancy at $T_{\mathrm{W}}$ for increasing $P$. Both mean field and simulations show that $\left|\mathrm{d} p_{\mathrm{B}} / \mathrm{d} T\right|$ displays a maximum that moves to lower $T$ for increasing $P$ (figure 5(d)). The temperature of this maximum coincides, within error bars, with $T_{\mathrm{W}}(P)$, consistent with the relation

$$
\alpha_{P}=\frac{v^{\prime}}{v} \alpha_{P}^{\prime}+2\left(\frac{v_{\mathrm{B}}}{v}\right)\left(\frac{\mathrm{d} p_{\mathrm{B}}}{\mathrm{d} T}\right)_{P},
$$

where $\alpha_{P}^{\prime}$ is the contribution arising from the fluctuations without taking into account the fluctuations due to the bonds. Moreover, $\left|\mathrm{d} p_{\mathrm{B}} / \mathrm{d} T\right|$ is related to the change of the local structure of the liquid, because it is proportional to the fluctuation of the number of bonds $N_{\mathrm{B}}$,

$$
\delta^{2} N_{\mathrm{B}} \equiv\left\langle N_{\mathrm{B}}^{2}\right\rangle-\left\langle N_{\mathrm{B}}\right\rangle^{2}=\frac{2 N k_{\mathrm{B}} T^{2}}{J-P v_{\mathrm{B}}}\left|\frac{\mathrm{d} p_{\mathrm{B}}}{\mathrm{d} T}\right| .
$$

Since the proportionality factor between $\left|\mathrm{d} p_{\mathrm{B}} / \mathrm{d} T\right|$ and $N_{\mathrm{B}}$ depends on $T$ and $P$, the locus of $\left|\mathrm{d} p_{\mathrm{B}} / \mathrm{d} T\right|^{\max }$ does not coincides with the locus of maximum fluctuations of $\left(\delta^{2} N_{\mathrm{B}}\right)^{\mathrm{max}}$, but the two loci approach each other for increasing $P$ and converge to $C^{\prime}$ (figure 5(a)). This result for the maximum fluctuation in the number of bonds, i.e. in the local structure, of bulk water in the vicinity of the Widom line on approaching $C^{\prime}$ is reminiscent of the observation of a maximum in structural fluctuations on crossing the Widom line in simulations for protein hydration water [43].

We find that $\left|\mathrm{d} p_{\mathrm{B}} / \mathrm{d} T\right|^{\max }$ increases on approaching $C^{\prime}$ in the same fashion as the response functions. Hence, for $P<P_{C^{\prime}}$, at $T>T_{\mathrm{W}}(P)$ the liquid has fewer bonds than for $T<T_{\mathrm{W}}(P)$, i.e., is less structured and more HDL-like, consistent with trends seen both in experiments [67] and in simulations $[43,73]$. For $J_{\sigma}>0$ the large fluctuations of $p_{\mathrm{B}}$ at $C^{\prime}$ shows that the LDL-HDL phase transition is a consequence of the cooperativity of the bonds due to the non-zero IM interaction [72].

\subsection{Dynamics in the supercritical region}

To study the dynamics, we calculate the relaxation time $\tau$ as the time for the spin autocorrelation function $C_{\sigma \sigma}(t) \equiv$ $\left\langle S_{i}(t) S_{i}(0)\right\rangle$ to decay to $1 / e$, where $S_{i} \equiv \sum_{j} \sigma_{i j} / 4$ quantifies the degree of total bond ordering for site $i$. The behavior of non-Arrhenius liquids can be represented by a Vogel-FulcherTamman (VFT) function

$$
\tau^{\mathrm{VFT}}=\tau_{0}^{\mathrm{VFT}} \exp \left[\frac{T_{1}}{T-T_{0}}\right],
$$



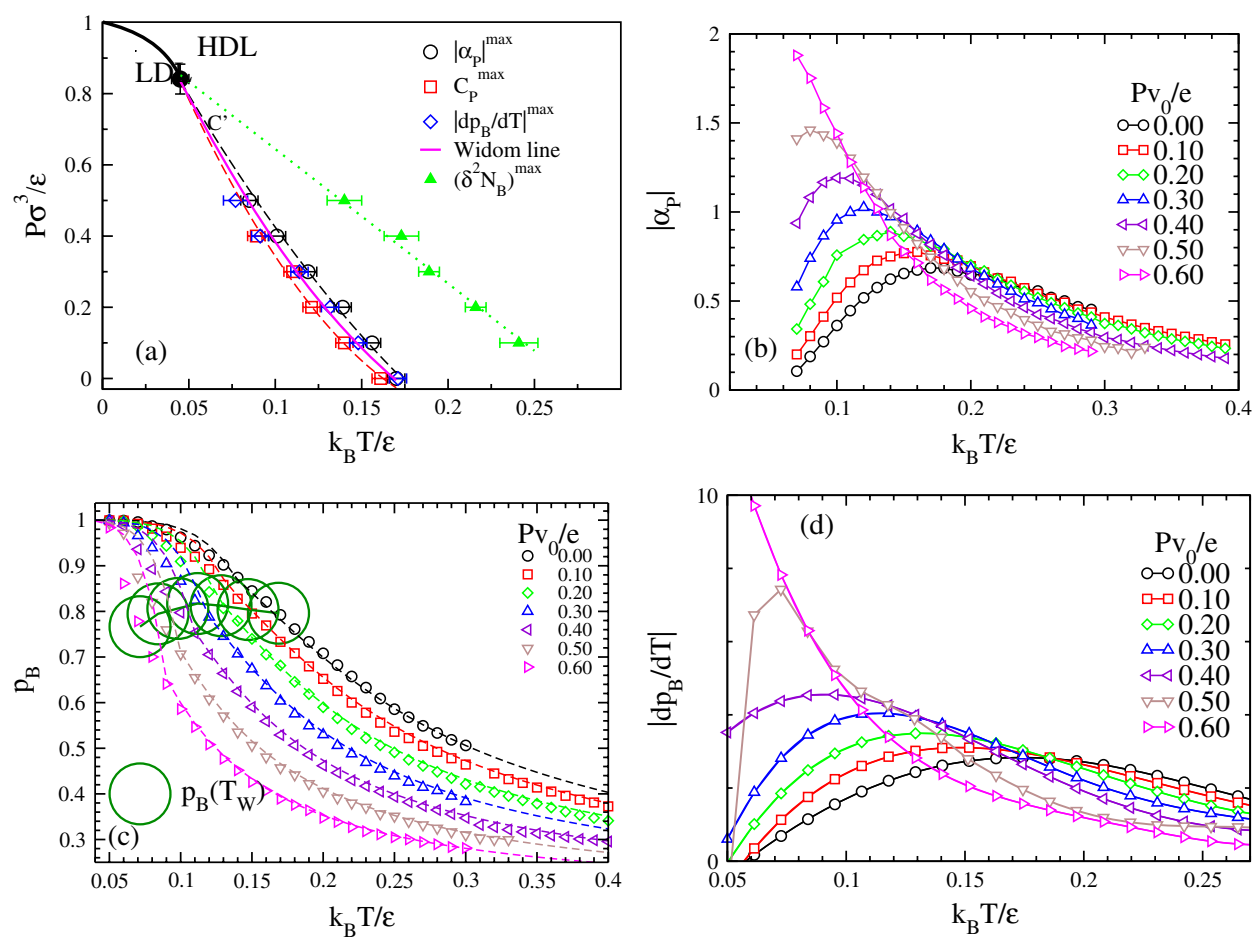

Figure 5. (a) The phase diagram below $T_{\mathrm{MD}}$ line for the water model with $J_{\sigma}>0$ : $C^{\prime}$ is the HDL-LDL critical point, end of first-order phase transition line (thick line) [66]; symbols are maxima for $N=3600$ of $\left|\alpha_{P}\right|^{\max }(\mathrm{O}), C_{P}^{\max }(\square),\left|\mathrm{d} p_{\mathrm{B}} / \mathrm{d} T\right|^{\max }(\diamond)$, and $\left(\delta^{2} N_{\mathrm{B}}\right)^{\max }(\triangle)$; our results show that $\left|\mathrm{d} p_{\mathrm{B}} / \mathrm{d} T\right|^{\max }$ coincides with the Widom line $T_{\mathrm{W}}(P)$ (solid line) within error bars; upper and lower dashed line are quadratic fits of $\left|\alpha_{P}\right|^{\max }$ and $C_{P}^{\max }$, respectively, merging at $P_{\mathrm{W}}^{\max }$; dotted line is crossing the symbols for $\left(\delta^{2} N_{\mathrm{B}}\right)^{\max }$ is a guide for the eyes; $\left|\alpha_{P}\right|^{\max }$ and $C_{P}^{\max }$ are consistent within error bars. Maxima are estimated from panels (b), (d) and 7(a), where each quantity is shown as functions of $T$ for different $P$. (b) The absolute value of the thermal expansion coefficient of $\alpha_{P}$ as a function of the temperature show a maximum for each pressure; lines are guides for the eyes. (c) The probability $p_{\mathrm{B}}$ of forming a bond (small symbols) increases for decreasing $T$, saturating to one at low $T$, with a larger increase at higher $P$; the value of $p_{\mathrm{B}}$ at $T_{\mathrm{W}}(P)$ is shown as a large open circle; the symbol has the size of the error on the $T_{\mathrm{W}}(P)$ estimate; dashed lines are the mean field calculations for $p_{\mathrm{B}}[71]$ and compare well with simulations for $T>T_{\mathrm{W}}(P)$ and $T \ll T_{\mathrm{W}}(P)$ with a discrepancy at $T \simeq T_{\mathrm{W}}(P)$ that is higher at higher $P$. (d) $\left|\mathrm{d} p_{\mathrm{B}} / \mathrm{d} T\right|^{\max }$ is the numerical derivative of $p_{\mathrm{B}}$ from simulations. In all the panels the errors, if not shown, are of the size of the symbols.
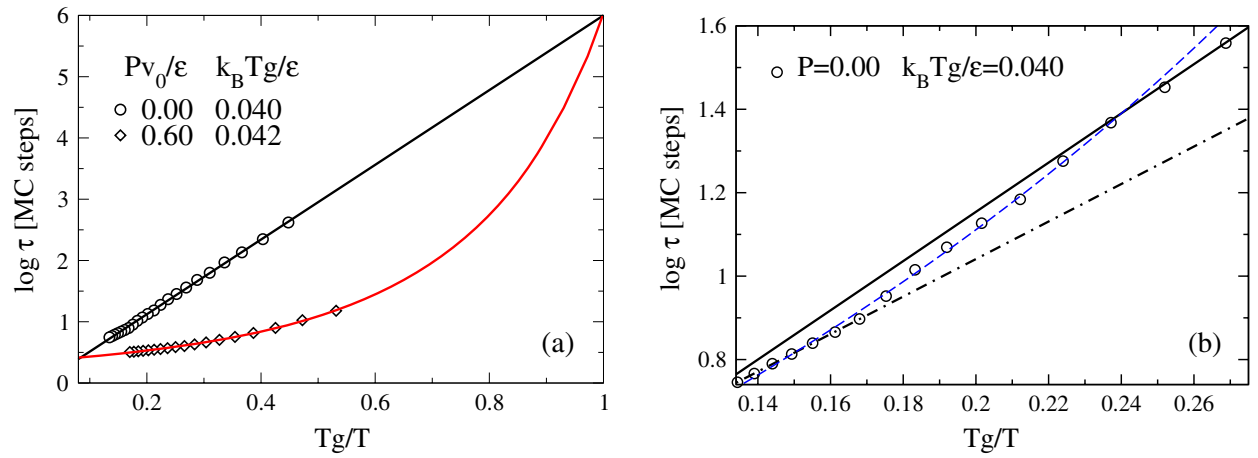

Figure 6. Angell's plot of the relaxation time $\tau$ at different pressures. $T_{\mathrm{g}}$ is a reference temperature at which the relaxation time is $\tau=10^{6}$ MC steps, arbitrarily used to rescale the temperatures. (a) At $P=0$ the behavior of the relaxation is apparently Arrhenius over all the range of explored temperatures; its behavior becomes increasingly non-Arrhenius upon increasing pressure, as shown for $P v_{0} / \epsilon=0.6$. (b) A closer look of the relaxation time at $P=0$ shows that it is non-Arrhenius at intermediate $T$, as in real water [74-76, 20].

where $\tau_{0}^{\mathrm{VFT}}, T_{1}$ and $T_{0}$ are all fitting parameters. Our results show that the liquid becomes more non-Arrhenius upon increasing $P$ (figure 6(a)).

For $P=0$, we show in figure 6(b) that upon decreasing $T$ there is a crossover from Arrhenius to VFT at intermediate temperatures, and then from VFT back to Arrhenius at lower $T$.
The Arrhenius activation energy at low $T$ is higher than that at high $T$, consistent with experiments at ambient $P$ for both bulk water [74, 75] and confined water [76, 20].

We find that for all $P$ the crossover occurs at $T_{\mathrm{W}}(P)$ within the error bars (figure 7(b)), confirming the idea proposed on the base of simulations of detailed models for water [34, 43]. 

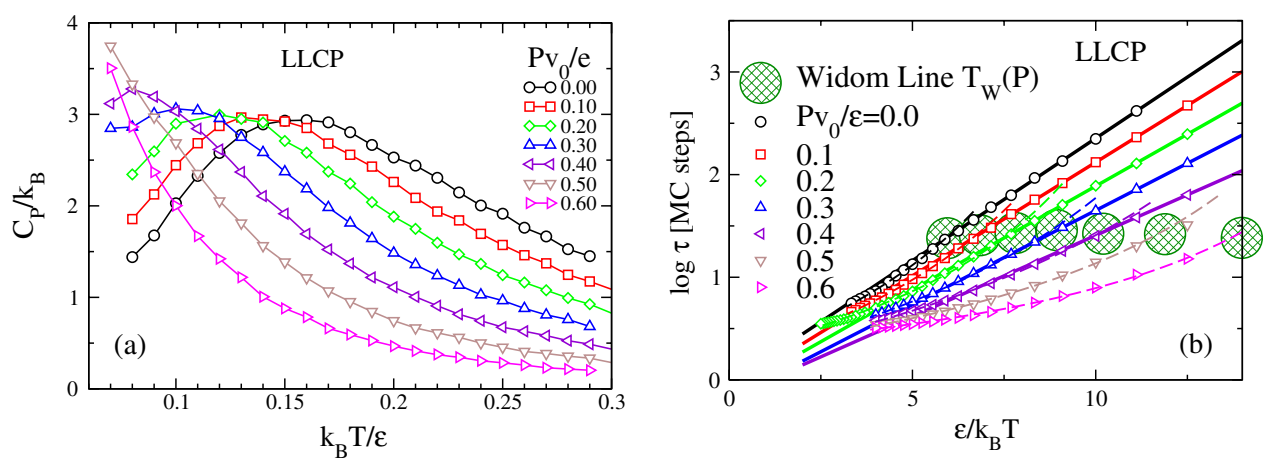

Figure 7. (a) Temperature dependence of specific heat $C_{P}$ for the LLCP scenario. $C_{P}$ has a maximum, the size of which increases with increasing pressure and diverges as $P \rightarrow P_{C^{\prime}}$. (b) Dynamic crossover in the LLCP case in the orientational relaxation time $\tau$ for a range of different pressures. The crossover occurs at temperature $T_{\mathrm{W}}(P)$ marked by large hatched circles of a radius approximately equal to the error bar on the estimate of $T_{\mathrm{W}}(P)$. Solid and dashed lines represent Arrhenius and VFT fits, respectively. The dynamic crossover occurs at approximately the same value of $\tau$ for all seven values of pressure studied.
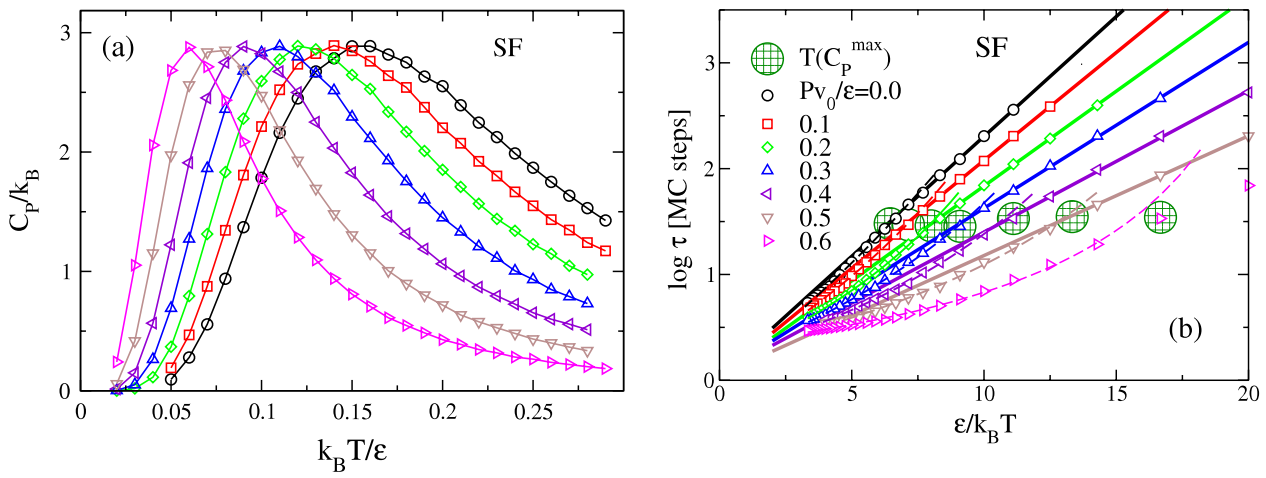

Figure 8. (a) Temperature dependence of specific heat $C_{P}$ for the $\mathrm{SF}$ scenario. $C_{P}$ has a maximum, but its size does not increase with increasing pressure, consistent with the findings of the mean field calculations of [45]. (b) Dynamic crossover in the SF scenario, with crossover temperature at $T\left(C_{P}^{\max }\right)$. Symbols are as in figure 7. Also in the SF scenario the dynamic crossover is isochronic.

We observe that the low- $T$ behavior is characterized by an activation energy - the slope in figure 7(b) — that decreases for increasing $P$, as in experiments for confined water [20].

Finally, we observe that the crossover is isochronic, i.e. the value of the crossover time $\tau_{\mathrm{C}}$ is approximately independent of pressure. We find $\tau_{\mathrm{C}} \simeq 10^{3 / 2} \mathrm{MC}$ steps $\simeq 15 \mathrm{ps}$ [17] (figure 7(b)). This means that the time needed to reach the maximum correlation length is almost independent of the position along $T_{\mathrm{W}}(P)$.

\section{Singularity-free (SF) scenario}

To further test whether the observed crossover is only a consequence of the liquid-liquid critical point, we also studied the dynamics in the $J_{\sigma}=0(\mathrm{SF})$ case.

\subsection{Fluctuations}

In the SF scenario the behavior of probability of forming bonds $p_{\mathrm{B}}$ is similar to that observed in the LLCP case, but $p_{\mathrm{B}}$ saturates to one at low $T$ at a lower rate with respect to the LLCP case $[45,72]$. Along the locus of maximum compressibility $K_{T}^{\max }, p_{\mathrm{B}}=0.795$ [45] is approximately equal to $p_{\mathrm{B}}\left(T_{\mathrm{W}}\right)$ observed along the Widom line in the LLCP scenario. This suggests that the structural behavior along the locus of maxima of the response functions, such as the compressibility or the specific heat, is independent on the presence of the LLCP $C^{\prime}$.

Both $K_{T}$ and $\left|\alpha_{P}\right|$ have maxima that increase along a line with negative slope in the $T-P$ phase diagram. This line coincides within the error bars to the locus of maxima of $C_{P}$. However, the $C_{P}$ maximum does not increase upon increasing pressure, but remains a constant (figure 8(a)), differently from the LLCP case.

\subsection{Dynamics}

We study the relaxation time $\tau$ of $C_{\sigma \sigma}(t)$ also for the SF scenario and we find that $\tau$ has a dynamic crossover similar to that seen in the LLCP case (figure 8(b)). As in the LLCP case, the dynamic crossover is isochronic with the crossover occurring at the same characteristic time $\tau_{\mathrm{C}} \simeq 10^{3 / 2} \mathrm{MC}$ steps (figure 8(b)).

Therefore, the presence of the dynamic crossover is consistent with both the SF and the LLCP scenario, leaving unclear if it is possible to distinguish between the two scenarios on the base of dynamic measurements. To clarify this point, 

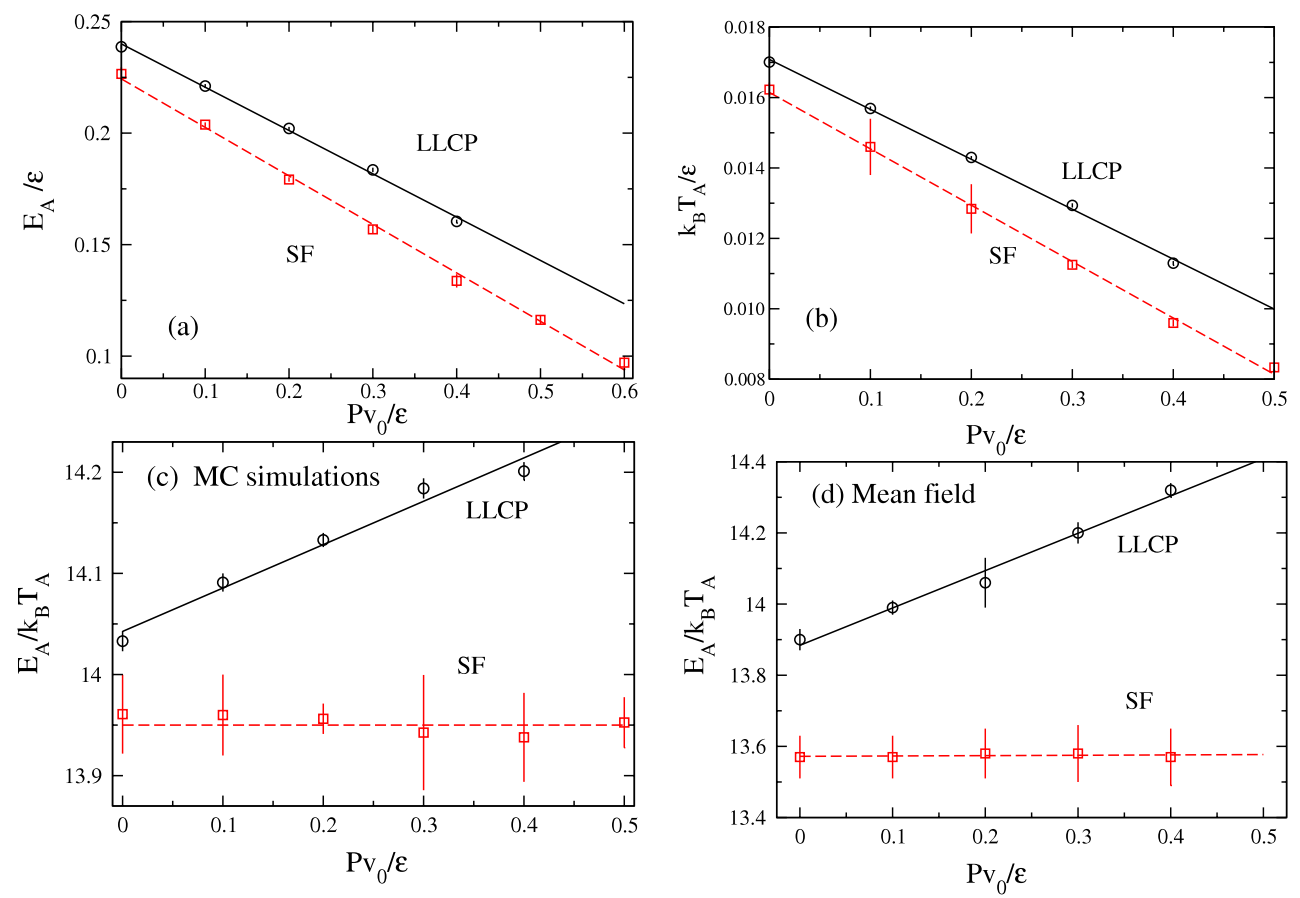

Figure 9. Effect of pressure on the activation energy $E_{\mathrm{A}}$. (a) Demonstration that $E_{\mathrm{A}}$ decreases linearly for increasing $P$ for both the LLCP and the SF scenarios. The lines are linear fits to the simulation results (symbols). (b) $T_{\mathrm{A}}$, defined such that $\tau\left(T_{\mathrm{A}}\right)=10^{14} \mathrm{MC}$ steps

$>100 \mathrm{~s}$ [17], decreases linearly with $P$ for both scenarios. (c) $P$ dependence of the quantity $E_{\mathrm{A}} /\left(k_{\mathrm{B}} T_{\mathrm{A}}\right)$ is different in the two scenarios. In the LLCP scenario, $E_{\mathrm{A}} /\left(k_{\mathrm{B}} T_{\mathrm{A}}\right)$ increases with increasing $P$, and it is approximately constant in the SF scenario. The lines are guides to the eyes. (d) Demonstration that the same behavior is found using the mean field approximation. In all the panels, where not shown, the error bars are smaller than the symbol sizes.

we investigate possible differences in the crossover for both scenarios [46].

\section{Difference in the pressure dependence of the dynamic crossover in LLCP and SF scenarios}

To see if there are any distinct differences in the pressure dependence of the dynamic crossover in the LLCP and SF case, we next calculate the Arrhenius activation energy $E_{\mathrm{A}}(P)$ from the low- $T$ slope of $\log \tau$ versus $1 / T$ (figure 9(a)).

\subsection{Monte Carlo simulations}

We extrapolate the temperature $T_{\mathrm{A}}(P)$ at which $\tau$ reaches a fixed macroscopic time $\tau_{\mathrm{A}} \geqslant \tau_{\mathrm{C}}$. We choose $\tau_{\mathrm{A}}=10^{14}$ MC steps $>100 \mathrm{~s}$, based on the observation that $1 \mathrm{MC}$ step $>\tau_{\alpha} \sim 10 \mathrm{ps}$, the $\alpha$-relaxation time in supercooled water, as results from the comparison with, e. g., [17] (figure 9(b)). We find that $E_{\mathrm{A}}(P)$ and $T_{\mathrm{A}}(P)$ decrease upon increasing $P$ in both scenarios, providing no distinction between the two interpretations. Instead, we find a dramatic difference in the $P$ dependence of the quantity $E_{\mathrm{A}} /\left(k_{\mathrm{B}} T_{\mathrm{A}}\right)$ in the two scenarios, increasing for the LLCP scenario and approximately constant for the SF scenario (figure 9(c)).

\subsection{Mean field analysis}

We can better understand our findings by developing an expression for $\tau$ in terms of thermodynamic quantities, which will then allow us to explicitly calculate $E_{\mathrm{A}} /\left(k_{\mathrm{B}} T_{\mathrm{A}}\right)$ for both scenarios. For any activated process, in which the relaxation from an initial state to a final state passes through an excited transition state, the relaxation time $\tau$ is related to the activation energy $\Delta(U+P V-T S)$, given by the difference in free energy between the transition state and the initial state, by the expression

$$
\ln \frac{\tau}{\tau_{0}}=\frac{\Delta(U+P V-T S)}{k_{\mathrm{B}} T},
$$

where $\tau_{0} \equiv \tau_{0}(P)$ is the relaxation time for $T \rightarrow \infty$.

Consistent with results from simulations and experiments [77, 78], we propose that at low $T$ the mechanism to relax from a less structured state (lower tetrahedral order) to a more structured state (higher tetrahedral order) corresponds to the breaking of a bond and the simultaneous molecular reorientation for the formation of a new bond. The transition state is represented by the molecule with a broken bond and more tetrahedral IM order. Hence,

$$
\Delta(U+P V-T S)=J p_{\mathrm{B}}-J_{\sigma} p_{\mathrm{IM}}-P v_{\mathrm{B}}-T \Delta S,
$$

where $p_{\mathrm{B}}$ and $p_{\mathrm{IM}}$, the probability of a satisfied IM interaction, can be directly calculated. To estimate $\Delta S$, the increase of entropy due to the breaking of a bond, we use the mean field expression

$$
\Delta S=k_{\mathrm{B}}\left[\ln \left(2 N p_{\mathrm{B}}\right)-\ln \left(1+2 N\left(1-p_{\mathrm{B}}\right)\right)\right] \bar{p}_{\mathrm{B}},
$$

where $\bar{p}_{\mathrm{B}}$ is the average value of $p_{\mathrm{B}}$ above and below $T_{\mathrm{W}}(P)$. 

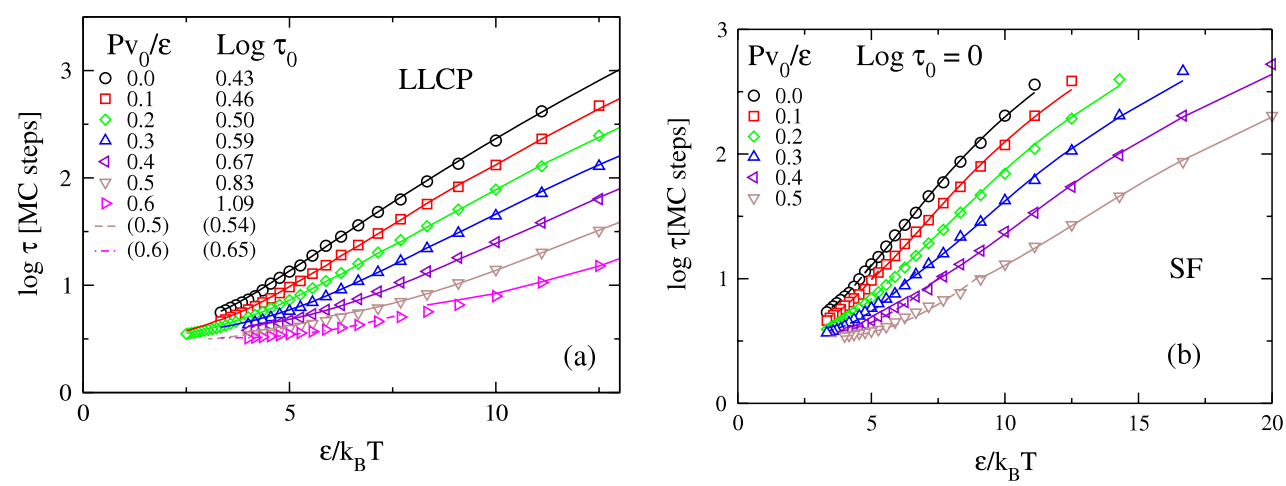

Figure 10. Comparison between mean field (lines) and Monte Carlo (symbols) calculations for the relaxation time $\tau$ for the LLCP (a) and SF (b) scenario. In (a) the fitting parameter $\log \tau_{0}$ for each pressure is given in the figures; dashed lines are the corrections at high $T$ for the equation (14); $\tau_{0}$ increases upon increasing $P$. In (b) $\log \tau_{0}=0$ and the equation (14) holds for all the pressures.

We next test if the expression of $\ln \left(\tau / \tau_{0}\right)$, in terms of $\Delta S$ and equation (12),

$$
\ln \frac{\tau}{\tau_{0}}=\frac{J p_{\mathrm{B}}-J_{\sigma} p_{\mathrm{IM}}-P v_{\mathrm{B}}}{k_{\mathrm{B}} T}-\bar{p}_{\mathrm{B}} \ln \frac{2 N p_{\mathrm{B}}}{1+2 N\left(1-p_{\mathrm{B}}\right)}
$$

describes the simulations well. Here $\tau_{0}$ is a free fitting parameter. We find that equation (14) holds over all the simulation range for the SF scenario and needs only minor corrections at high $T$ and $P$ for the LLCP case (figure 10). The corrections are expected since we are considering a relaxation process that has larger probability at low $T$ and $P$.

In the SF case $\tau_{0}=1 \mathrm{MC}$ step for any $P$, while in the LLCP case $\tau_{0}$ increases with $P$. This is consistent with the fact that at high pressure and temperatures, when there are no hydrogen bonds, water behaves like a normal liquid, which has larger relaxation times for higher densities.

From equation (14) we find that the ratio $E_{\mathrm{A}} /\left(k_{\mathrm{B}} T_{\mathrm{A}}\right)$ calculated at low $T$ increases with $P$ for $J_{\sigma} / \epsilon=0.05$, while it is constant for $J_{\sigma}=0$, as from our simulations (figure $9(\mathrm{~d})$ ). Therefore, the mean field analysis is able to rationalize our simulation results.

\section{Summary}

Simulations of bulk supercooled water, as those for protein hydration water, show a crossover from non-Arrhenius to Arrhenius dynamics of relaxation time of the hydrogen bonds. Our study show that:

- The dynamic crossover is consistent with both the LLCP and the SF scenarios. The crossover occurs at a temperature close to $T\left(C_{P}^{\max }\right)$, which decreases for increasing $P$.

- Our mean field analysis allows us to rationalize the dynamic crossover as a consequence of a local breaking and reorientation of the bonds for the formation of new and more tetrahedrally oriented bonds. Above $T\left(C_{P}^{\max }\right)$, when $T$ decreases, the number of hydrogen bonds increases, giving rise to an increasing activation energy $E_{\mathrm{A}}$ and to a non-Arrhenius dynamics. As $T$ decreases, entropy must decrease. A major contributor to entropy is the orientational disorder, that is a function of the probability $p_{\mathrm{B}}$ of forming bonds, as described by the mean field expression for $\Delta S$ equation (13).

- We find that, as $T$ decreases, $p_{\mathrm{B}}$-hence the orientational order-increases. We find that the rate of increase has a maximum at $T\left(C_{P}^{\max }\right)$, and as $T$ continues to decrease this rate drops rapidly to zero-meaning that for $T<$ $T\left(C_{P}^{\max }\right)$, the local orientational order rapidly becomes temperature independent and the activation energy $E_{\mathrm{A}}$ also becomes approximately temperature independent, for the equation (12). Corresponding to this fact the dynamics becomes approximately Arrhenius.

- We find that the crossover is approximately isochronic (independent of the pressure) consistent with our calculations of an almost constant number of bonds at $T\left(C_{P}^{\max }\right)$.

- We observe that in both scenarios the Arrhenius activation energy $E_{\mathrm{A}}$ and the temperature $T_{\mathrm{A}}$, at which the relaxation time is macroscopic, decrease upon increasing $P$. Instead, the $P$ dependence of the quantity $E_{\mathrm{A}} /\left(k_{\mathrm{B}} T_{\mathrm{A}}\right)$ has a dramatically different behavior in the two scenarios. For the LLCP scenario it increases as $P \rightarrow P_{C^{\prime}}$, while it is approximately constant in the SF scenario. Therefore, the quantity $E_{\mathrm{A}} /\left(k_{\mathrm{B}} T_{\mathrm{A}}\right)$ offers a means to distinguish between the two interpretations by dynamic measurements.

\section{Acknowledgments}

We thank C A Angell, W Kob, and S Sastry for helpful discussions and NSF grant CHE 0616489 for support. GF also thanks the Spanish Ministerio de Educación y Ciencia (Programa Ramón y Cajal and Grant No. FIS2007-61433).

\section{References}

[1] Debenedetti P G 2003 J. Phys.: Condens. Matter 15 R1669 Debenedetti P G and Stanley H E 2003 Phys. Today 5640

[2] Marion G M and Jakubowski S D 2004 Cold regions Sci. Technol. 38211

[3] Poole P H et al 2005 J. Phys.: Condens. Matter 17 L431 
[4] Mallamace F, Broccio M, Corsaro C, Faraone A, Wanderlingh U, Liu L, Mou C-Y and Chen S H 2006 J. Chem. Phys. 124161102

[5] Liu D, Zhang Y, Chen C-C, Mou C-Y, Poole P H and Chen S-H 2007 Proc. Natl Acad. Sci. 1049570

Mallamace F, Branca C, Broccio M, Corsaro C, Mou C-Y and Chen S-H 2007 Proc. Natl Acad. Sci. USA 10418387

[6] Stanley H E 1979 J. Phys. A 12 L329

[7] Huang K 2001 Introduction to Statistical Physics (London: Taylor and Francis)

[8] Angell C A, Shuppert J and Tucker J C 1973 J. Phys. Chem. 77 3092-9

Speedy R J and Angell C A 1976 J. Chem. Phys. 65 851-8

[9] Bett K E and Cappi J B 1965 Nature 207620

DeFries T and Jonas J 1977 J. Chem. Phys. 66896

[10] Angell C A, Finch E D and Bach P 1976 J. Chem. Phys. $\mathbf{6 5} 3065$

[11] Berendsen H J C, Grigera J R and Straatsma T P 1987 J. Phys. Chem. 916269

[12] Starr F W, Sciortino F and Stanley H E 1999 Phys. Rev. E 606757

[13] Errington J R and Debenedetti P G 2001 Nature 409318

[14] Netz P A, Starr F W, Stanley H E and Barbosa M C 2001 J. Chem. Phys. 115344

[15] Mudi A, Chakravarty C and Ramaswamy R 2005 J. Chem. Phys. 122104507

[16] Mittal J, Errington J R and Truskett T M 2006 J. Phys. Chem. B 11018147

[17] Kumar P, Franzese G, Buldyrev S V and Stanley H E 2006 Phys. Rev. E 73041505

[18] Angell C A 2008 Science 319582

[19] Crupi V, Magazu S, Majolino D, Migliardo P, Venuti V and Bellissent-Funel M-C 2000 J. Phys.: Condens. Matter 123625

[20] Liu L et al 2005 Phys. Rev. Lett. 95117802

[21] Swenson J, Jansson H and Bergman R 2006 Phys. Rev. Lett. 96247802

[22] Chen S-H, Liu L, Fratini E, Baglioni P, Faraone A and Mamontov E 2006 Proc. Natl Acad. Sci. USA 1039012

[23] Bellissent-Funel M-C 2000 J. Mol. Liq. 8439

[24] Koga K, Zeng X C and Tanaka H 1997 Phys. Rev. Lett. 795262 Koga K, Zeng X C and Tanaka H 1998 Chem. Phys. Lett. $285278-83$

[25] Meyer M and Stanley H E 1999 J. Phys. Chem. B 1039728

[26] Zangi R and Mark A E 2003 J. Chem. Phys. 1191694 Zangi R 2004 J. Phys.: Condens. Matter 16 S5371

[27] Kumar P, Buldyrev S V, Starr F W, Giovambattista N and Stanley H E 2005 Phys. Rev. E 72051503

Kumar P, Starr F W, Buldyrev S V and Stanley H E 2007 Phys. Rev. E 75011202

[28] Gallo P 2000 Phys. Chem. Phys. 21607

Gallo P, Rovere M, Ricci M A, Hartnig C and Spohr E 2000 Europhys. Lett. 49 183-8

Gallo P and Rovere M 2007 Phys. Rev. E 76061202

[29] Giovambattista N, Rossky P J and Debenedetti P G 2006 Phys. Rev. E 73041604

[30] Marty J, Nagy G and Gordillo M C 2006 J. Chem. Phys. 124094703

[31] Brovchenko I and Oleinikova A 2007 J. Chem. Phys. 126214701

[32] Chu X-Q, Kolesnikov A I, Moravsky A P, Garcia-Sakai V and Chen S-H 2007 Phys. Rev. E 76021505

[33] Han S, Kumar P and Stanley H E 2008 Phys. Rev. E 77030201

[34] Xu L, Kumar P, Buldyrev S V, Chen S-H, Poole P H, Sciortino F and Stanley H E 2005 Proc. Natl Acad. Sci. 10216558

[35] Poole P H, Sciortino F, Essmann U and Stanley H E 1992 Nature $\mathbf{3 6 0} 324$
Mishima O and Stanley H E 1998 Nature 392164

[36] Jagla E A 2001 Phys. Rev. E 63061501

[37] Buldyrev S V, Franzese G, Giovambattista N, Malescio G, Sadr-Lahijany M R, Scala A, Skibinsky A and Stanley H E 2002 Physica A $\mathbf{3 0 4} 23$

[38] Franzese G 2007 J. Mol. Liq. 136267

[39] Yan Z, Buldyrev S V, Giovambattista N and Stanley H E 2005 Phys. Rev. Lett. 95130604

Xu L, Buldyrev S, Angell C A and Stanley H E 2006 Phys. Rev. E 74031108

[40] de Oliveira A B, Netz P A, Colla T and Barbosa M C 2006 J. Chem. Phys. 124084505

de Oliveira A B, Netz P A, Colla T and Barbosa M C 2006 J. Chem. Phys. 125124503

[41] de Oliveira A B, Franzese G, Netz P A and Barbosa M C 2008 J. Chem. Phys. 128064901

[42] Franzese G, Malescio G, Skibinsky A, Bulderev S V and Stanley H E 2001 Nature 409692

Franzese G, Malescio G, Skibinsky A, Bulderev S V and Stanley H E 2002 Phys. Rev. E 66051206

Malescio G, Franzese G, Pellicane G, Skibinsky A, Buldyrev S V and Stanley H E 2002 J. Phys.: Condens. Matter 142193

Skibinsky A, Buldyrev S V, Franzese G, Malescio G and Stanley H E 2004 Phys. Rev. E 69061206

Malescio G, Franzese G, Skibinsky A, Buldyrev S V and Stanley H E 2005 Phys. Rev. E 71061504

[43] Kumar P, Yan Z, Xu L, Mazza M, Buldyrev S V, Chen S-H, Sastry S and Stanley H E 2006 Phys. Rev. Lett. 97177802

[44] Kumar P, Buldyrev S V, Becker S L, Poole P H, Starr F W and Stanley H E 2007 Proc. Natl Acad. Sci. 1049575

[45] Stanley H E and Teixeira J 1980 J. Chem. Phys. 733404

Sastry S, Debenedetti P G, Sciortino F and Stanley H E 1996 Phys. Rev. E 536144

[46] Kumar P, Franzese G and Stanley H E 2008 Phys. Rev. Lett. 100105701

[47] Rebelo L P N, Debenedetti P G and Sastry S 1998 J. Chem. Phys. 109106

[48] Maruyama S, Wakabayashi K and Oguni M 2004 AIP Conf. Proc. 708675

[49] Zanotti J M, Bellissent-Funel M-C and Parrello J 1999 Biophys. J. 762390

[50] Ringe D and Petsko G A 2003 Biophys. Chem. 105667

[51] Wang J, Cieplak P and Kollman P A 2000 J. Comput. Chem. 211049

[52] Rasmussen B F, Ringe M and Petsko G A 1992 Nature 357423

[53] Vitkup D, Ringe D, Petsko G A and Karplus M 2000 Nat. Struct. Biol. 734

[54] Sokolov A P, Grimm H, Kisliuk A and Dianoux A J 1999 J. Chem. Phys. 1107053

[55] Sorin E J and Pande V S 2005 Biophys. J. 882472

[56] Doster W, Cusack S and Petry W 1989 Nature 338754

[57] Norberg J and Nilsson L 1996 Proc. Natl Acad. Sci. USA 9310173

[58] Tarek M and Tobias D J 2002 Phys. Rev. Lett. 88138101 Tarek M and Tobias D J 2000 Biophys. J. 793244

[59] Hartmann H, Parak F, Steigemann W, Petsko G A, Ponzi D R and Frauenfelder H 1982 Proc. Natl Acad. Sci. USA 794067

[60] Tournier A L, Xu J and Smith J C 2003 Biophys. J. 851871

[61] Lee A L and Wand A J 2001 Nature 411501

[62] Lindahl E, Hess B and van der Spoel D $2001 \mathrm{~J}$. Mol. Modeling 7306

[63] Artymiuk P J, Blake C C F, Rice D W and Wilson K S 1982 Acta Crystallogr. B $\mathbf{3 8} 778$ 
[64] Drew H R, Wing R M, Takano T, Broka C, Tanaka S, Itakura K and Dickerson R E 1981 Proc. Natl Acad. Sci. USA 782179

[65] Stanley H E, Buldyrev S V, Franzese G, Giovambattista N and Starr F W 2005 Phil. Trans. R. Soc. 363509

[66] Franzese G and Stanley H E 2002 Physica A 314508 Franzese G and Stanley H E 2002 J. Phys.: Condens. Matter 142201

Franzese G, Marqués M I and Stanley H E 2003 Phys. Rev. E 67011103

[67] D'Arrigo G, Maisano G, Mallamace F, Migliardo P and Wanderlingh F 1981 J. Chem. Phys. 754264

Angell C A and Rodgers V 1984 J. Chem. Phys. 806245

[68] Soper A K and Ricci M A 2000 Phys. Rev. Lett. 842881 and references cited therein

[69] Schwegler E, Galli G and Gygi F 2000 Phys. Rev. Lett. 842429
Raiteri P, Laio A and Parrinello M 2004 Phys. Rev. Lett. 93087801 and references cited therein

[70] Franzese G, Cataudella V, Korshunov S E and Fazio R 2000 Phys. Rev. B 62 R9287

[71] Franzese G and Stanley H E 2007 J. Phys.: Condens. Matter 19205126

[72] Stokely K, Mazza M, Franzese G and Stanley H E 2008 in preparation

[73] Paschek D 2005 Phys. Rev. Lett. 94217802

Yamada M, Mossa S, Stanley H E and Sciortino F 2002 Phys. Rev. Lett. 88195701

[74] Ito K, Moynihan C T and Angell C A 1999 Nature 398492

[75] Kohl I, Bachmann L, Hallbrucker A, Mayer E and Loerting T 2005 Phys. Chem. Chem. Phys. 73210

[76] Bergman R and Swenson J 2000 Nature 403283

[77] Laage D and Hynes J T 2006 Science 311832

[78] Tokmakoff A 2007 Science $\mathbf{3 1 7} 54$ and references cited therein 